\title{
Die Tracheotomie auf der Intensivstation
}

\section{Tracheostomy in the Intensive Care Unit}

Autoren

Institute
H. J. Baumann ${ }^{1,2}$, C. Kemei ${ }^{1}$, S. Kluge

Klinik für Intensivmedizin, Universitätsklinikum Hamburg-Eppendorf

Sektion Pneumologie, II. Medizinische Klinik, Universitätsklinikum Hamburg-Eppendorf eingereicht 9.8.2010

akzeptiert $\quad 16.8 .2010$

\section{Bibliografie}

Dol http://dx.doi.org/ 10.1055/s-0030-1255743

Online-Publikation: 20. 9. 2010

Pneumologie 2010; 64:

769-776 @ Georg Thieme

Verlag KG Stuttgart · New York

ISSN 0934-8387

Korrespondenzadresse

PD Dr. med. Stefan Kluge

Klinik für Intensivmedizin

Universitätsklinikum

Hamburg-Eppendorf

Martinistraße 52

20246 Hamburg

skluge@uke.de

Serienherausgeber

S. Rosseau, Berlin

B. Schönhofer, Hannover

\section{Zusammenfassung \\ $\nabla$}

In den letzten Jahren ist es im Bereich der Intensivmedizin zu einem erheblichen Wandel hinsichtlich Indikation, Zeitpunkt und Technik der Tracheotomie gekommen. Die perkutane Dilatationstracheotomie hat sich hierbei zu einem Standardverfahren entwickelt. Sie wird im Regelfall durch den Intensivmediziner selbst durchgeführt und weist bei entsprechender Sorgfalt eine niedrige Komplikationsrate auf. Vorteile dieser Technik gegenüber der chirurgischen Technik sind die geringe Inzidenz von Stomainfektionen, das bessere kosmetische Ergebnis nach Dekanülierung, fehlende Notwendigkeit eines operativen Stomaverschlusses sowie eine Kostenersparnis. Essentiell bei der dilatativen Technik sind die Kenntnis der Anatomie, die Beachtung der Kontraindikationen und eine durchgehende bronchoskopische Kontrolle während der Tracheotomie. Zudem erfordert der Kanülenwechsel bei dilatativ angelegtem Tracheostoma spezielle Kenntnisse und Vorbereitungen. Bestehen Kontraindikationen für eine perkutane Dilatationstracheotomie, so ist eine chirurgische Tracheotomie indiziert. Der optimale Tracheotomiezeitpunkt wird weiterhin kontrovers diskutiert.

\section{Einleitung}

\section{$\nabla$}

Der chirurgische Eingriff einer Tracheotomie gehört zu den ältesten chirurgischen Verfahren der Medizin. Schon im heiligen Buch der hinduistischen Medizin, dem Rigveda (ca. 2000 v.Chr.), sowie in alten ägyptischen Schriften (ca. 1500 v.Chr.) finden sich Erörterungen über die Einführung einer Kanüle in die Trachea [1].

Waren in den vergangenen Jahrhunderten insbesondere entzündliche Prozesse und Fremdkörper Hauptindikationen für eine Tracheotomie, so sind es heute die Anlage passagerer oder dauerhafter Stomata bei Tumoroperationen im Kopf-Hals-Be-

\section{Abstract \\ $\nabla$}

Indication, timing and technique of tracheostomy have undergone considerable changes during the past years. Percutaneous tracheostomy has become a standard procedure in modern intensive care. It can be performed by the intensive medicine specialists themselves with low complication rates. Advantages are less stomal infections, better cosmetic results and cost savings in comparison to the surgical technique. Exact knowledge of the anatomic details, careful attention to contraindications and bronchoscopic supervision throughout the procedure are essential for optimal results. Exchange of the tracheostomy tube requires adequate preparation and training. If contraindications exist or permanent mechanical ventilation can be anticipated, surgical tracheostomy is indicated. The optimal timing of tracheostomy remains controversial.

reich und die Langzeitbeatmung des Intensivpatienten. Mit der Entwicklung der modernen Intensivmedizin und der steigenden Zahl von langzeitbeatmeten Patienten wurde die Tracheotomie zunehmend Bestandteil der Beatmungstherapie. Hauptziele der Tracheotomie beim Intensivpatienten sind die Vermeidung von Kehlkopfschäden, die Erleichterung der Pflege des Nasen-Rachen-Bereiches [2] und das Schaffen eines sicheren Beatmungszugangs bei kritischem Gasaustausch oder notwendigen Lagerungsmanövern. Durch die nichtinvasive Beatmung kann heutzutage, z. B. bei akuter respiratorischer Insuffizienz auf dem Boden einer exazerbierten COPD oder 

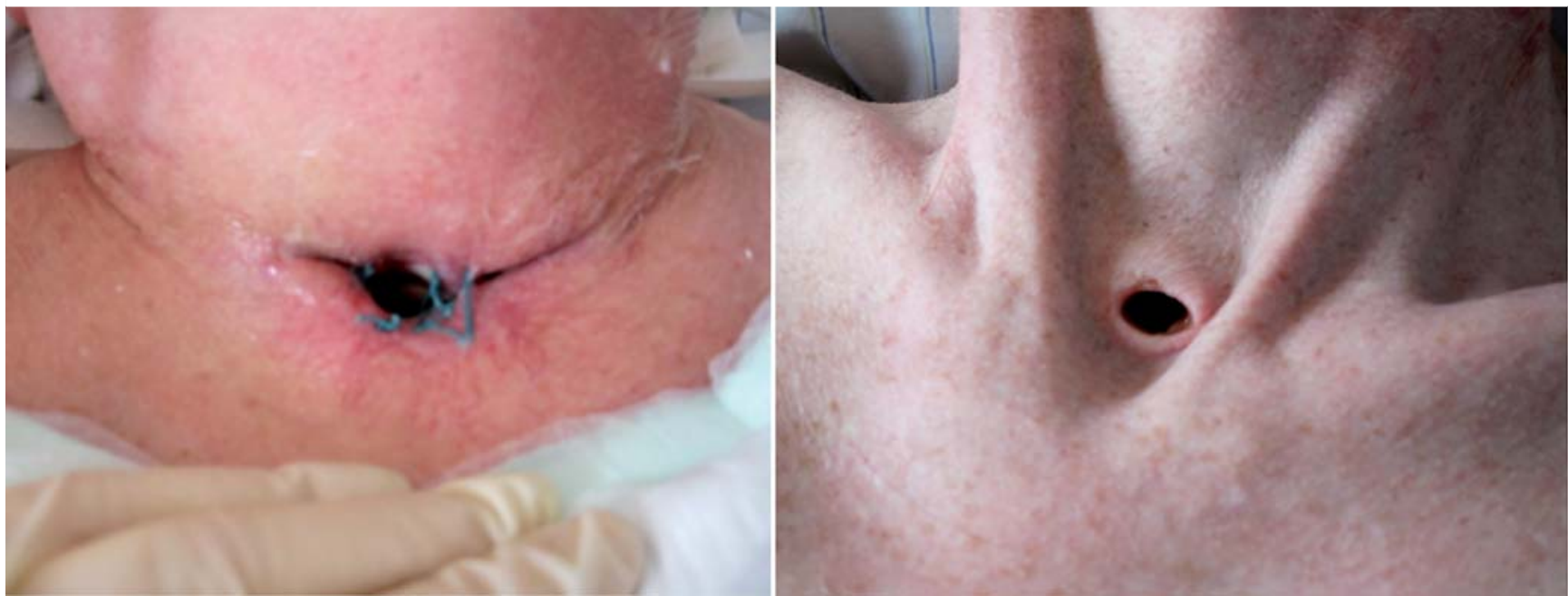

Abb. 1 Vergleich der Techniken. links konventionelles („chirurgisches“) Stoma; rechts dilatatives Stoma.

beim kardiogenen Lungenödem, bei einem Teil der Patienten auf eine Intubation und invasive Beatmung verzichtet werden. Trotzdem ist es in den letzten Jahren zu einer deutlichen Zunahme von Tracheotomien auf den Intensivstationen gekommen [3]. Gründe dafür sind zum einen die generellen Fortschritte in der intensivmedizinischen Behandlung Schwerstkranker mit einer damit einhergehenden zunehmenden Anzahl von langzeitbeatmeten Patienten, zum anderen zeigen Studien der letzten Jahre, dass bei bestimmten Patientenkollektiven eine frühzeitige Tracheotomie die Zeitdauer der Beatmung und den Intensivaufenthalt verkürzt [4].

In den letzten zweieinhalb Jahrzehnten wurden alternativ zur klassischen chirurgischen Tracheotomietechnik verschiedene minimal-invasive Techniken, sogenannte perkutane Dilatationstracheotomien, zur Minimierung des Gewebstraumas entwickelt (๑ Abb. 1).

Unter einer perkutanen Dilatationstracheotomie wird eine Tracheotomie verstanden, bei der nach perkutaner Nadelpunktion der Trachea und Platzierung eines Führungsdrahtes ein Tracheostoma mit Hilfe von Dilatationsinstrumenten angelegt wird. Seit der Einführung der modernen perkutanen Dilatationstracheotomie im Jahre 1985 durch den amerikanischen Chirurgen Pasquale Ciaglia [5] folgten inzwischen fünf weitere Verfahren.

Die dilatative Technik, welche im Regelfall durch den Intensivmediziner selbst durchgeführt wird und niedrige Komplikationsraten aufweist, hat sich hierbei in den letzten Jahren zu einem Standardverfahren auf den Intensivstationen entwickelt. Insgesamt hat sich hinsichtlich der Indikation, des Zeitpunktes und der Technik der Tracheotomie in der Intensivmedizin in den letzten Jahren ein deutlicher Wandel ergeben. Der vorliegende Übersichtsartikel soll den aktuellen Stand der Tracheotomie auf der Intensivstation darstellen.

\section{Indikation zur Tracheotomie \\ $\nabla$}

Die Hauptindikation für eine Tracheotomie beim Intensivpatienten ist die Langzeitbeatmung. Im Rahmen einer persistierenden Sekretretention kann - besonders bei chronisch ateminsuffizienten und/oder neurologisch erkrankten Patienten - eine Tracheotomie als direkter Zugang für die tracheale Sekretabsaugung indiziert sein, wenn andere Sekreteliminationstechniken nicht er- folgreich sind. Selten wird eine Tracheotomie aufgrund von Stenosen der oberen Atemwege, Tumoren oder Verletzungen im Mund- oder Rachenbereich beim Intensivpatienten erforderlich sein. In der Vergangenheit war eines der Hauptargumente für die Tracheotomie beim Intensivpatienten die Vermeidung von Larynxschäden durch den Endotrachealtubus ( $\bullet$ Tab. 1).

Aufgrund verbesserter Tubuseigenschaften ist diese Problematik in den letzten Jahrzehnten etwas in den Hintergrund getreten, allerdings ist die Dauer der invasiven Beatmung nach wie vor der wichtigste Faktor für die Entwicklung laryngotrachealer Schäden [6].

Angeführte atemphysiologische Argumente für die Erleichterung des Weanings durch eine Tracheotomie im Vergleich zur fortgesetzten endotrachealen Intubation sind u. a. ein reduziertes Totraumvolumen, eine Verringerung des Atemwegswiderstandes sowie der Atemarbeit [7]. Allerdings sind die Effekte nur gering. So betrug die Totraumdifferenz in der Studie von Davis et al [8] weniger als $20 \mathrm{ml}$. Zu beachten ist, dass Atemwegswiderstand und Atemarbeit stark von der Größe der gewählten Trachealkanüle abhängig sind.

Die bessere Toleranz einer Trachealkanüle im Vergleich zum translaryngealen Endotrachealtubus erlaubt im Regelfall die Reduktion von Sedativa und Analgetika. Der Wegfall eines Hindernisses im Mundraum ermöglicht zudem eine bessere Pflege des Nasen-Rachen-Bereiches, zudem wird die Bronchialtoilette erleichtert. All diese Faktoren tragen zu einem erhöhten Patientenkomfort bei [9]. Durch spezielle Sprechkanülen wird die Kommunikation gefördert, zusätzlich kann die Mimik des Patienten wieder gelesen werden [10]. Einige Studien berichten über eine

Tab. 1 Vorteile der Tracheotomie gegenüber der Langzeitintubation.

Verminderung bzw. Vermeidung von Larynxschäden wie Luxationen, Ulzerationen und Nekrosen der Aryknorpel

Verringerung von Atemarbeit und Atemwegswiderstand

Reduktion des Bedarfs an Analgetika und Sedativa

Erhöhung des Patientenkomforts

Möglichkeit der Kommunikation durch Sprechkanülen oder Aufsätze, Lesen der Mimik

Erleichterung der Bronchialtoilette, verbesserte Pflege des Nasen-

Rachenraumes

Orale Ernährung möglich (Schluckvorgang möglich) 
geringere Inzidenz von ventilatorassoziierten Pneumonien (VAP) bei tracheotomierten Patienten verglichen mit endotrachealer Intubation [11,12]. Dieser Effekt ist jedoch nur inkonsistent nachweisbar und erfordert weitere Studien zu diesem Thema [10].

\section{Die perkutane Dilatationstracheotomie \\ $\nabla$}

\section{Verfahren}

Der amerikanische Chirurg Pasquale Ciaglia beschrieb 1985 eine Tracheotomiemethode mit Bougierung des Tracheostomas durch mehrere, im Durchmesser zunehmende Kunststoffdilatatoren (klassische Ciaglia-Technik) und begründete damit die Ära der modernen perkutanen Dilatationstracheotomie [5].

Wenige Jahre später entwickelte der Australier William M. Griggs die GWDF-Technik (Guide Wire Dilating Forceps) [13], bei der die Dilatation mit einer Spreizzange erfolgt. Die Spreizzange wird hierbei über eine zentrale Bohrung auf den in der Trachea liegenden Seldingerdraht aufgefädelt. Eine weitere Technik mit retrograder Einführung der Trachealkanüle (TLT) wurde 1997 von Antonio Fantoni eingeführt [14]. Bei dieser Technik wird eine spezielle Trachealkanüle über den aus dem Mund austretenden Seldingerdraht gefädelt, welche dann, durch Zug am halsseitigen Drahtende, von innen durch die Punktionsstelle nach außen durchgezogen wird. In Abwandlung der Methode nach Ciaglia wurde mit der Ciaglia Blue Rhino ${ }^{\circledR}$-Methode [15] ein Einschrittverfahren mit nur noch einem konisch geformten Dilatator entwickelt. Der mehrfache Dilatatorenwechsel entfällt somit. 2002 stellten Giulio Frova und Michael Quintel [16] die PercuTwist ${ }^{\circledR}$ Technik vor. Die Dilatation erfolgt hierbei mit einer über den Seldingerdraht eingebrachten selbstschneidenden Dilatationsschraube. Zuletzt wurde 2005 die Blue-Dolphin ${ }^{\circledR}$-Methode beschrieben, bei der die Dilatation radiär mittels eines Ballons erfolgt [17].

Derzeit stehen somit sechs Methoden der perkutanen Dilatationstracheotomie zur Verfügung. Allen Techniken gemeinsam ist die Punktion der Trachea mit einer Kanüle ( $\bullet$ Abb. 2), über die ein Draht in Seldinger-Technik eingeführt wird, die Punktionskanüle entfernt und mittels Dilatatoren das prätracheale Gewebe eröffnet wird.

Die unterschiedlichen dilatativen Verfahren wurden in diversen Studien verglichen. Dabei zeigte sich, dass die Rate der klinisch relevanten Komplikationen für alle Verfahren gleichermaßen niedrig ist [18]. Letztendlich bleibt es dem Arzt überlassen, welche Technik angewendet wird. Die Anwendung von mehr als zwei dilatativen Methoden erscheint jedoch nicht sinnvoll. Vielmehr sollte eine (Haupt)-Technik nach persönlicher Neigung ausgewählt und diese dann durch regelmäßige Anwendung perfektioniert werden.

Eine aktuelle Umfrage auf 447 deutschen Intensivstationen zeigt, dass $86 \%$ der Befragten routinemäßig dilatative Tracheotomien durchführen, 73\% der Ärzte gaben an, die perkutane Dilatationstracheotomie als Tracheotomieverfahren der ersten Wahl bei langzeitbeatmeten Patienten anzusehen. Unter den verschiedenen Techniken wird die Ciaglia Blue Rhino ${ }^{\circledR}$-Technik am häufigsten verwendet, gefolgt von der GWDF-Methode nach Griggs [19].
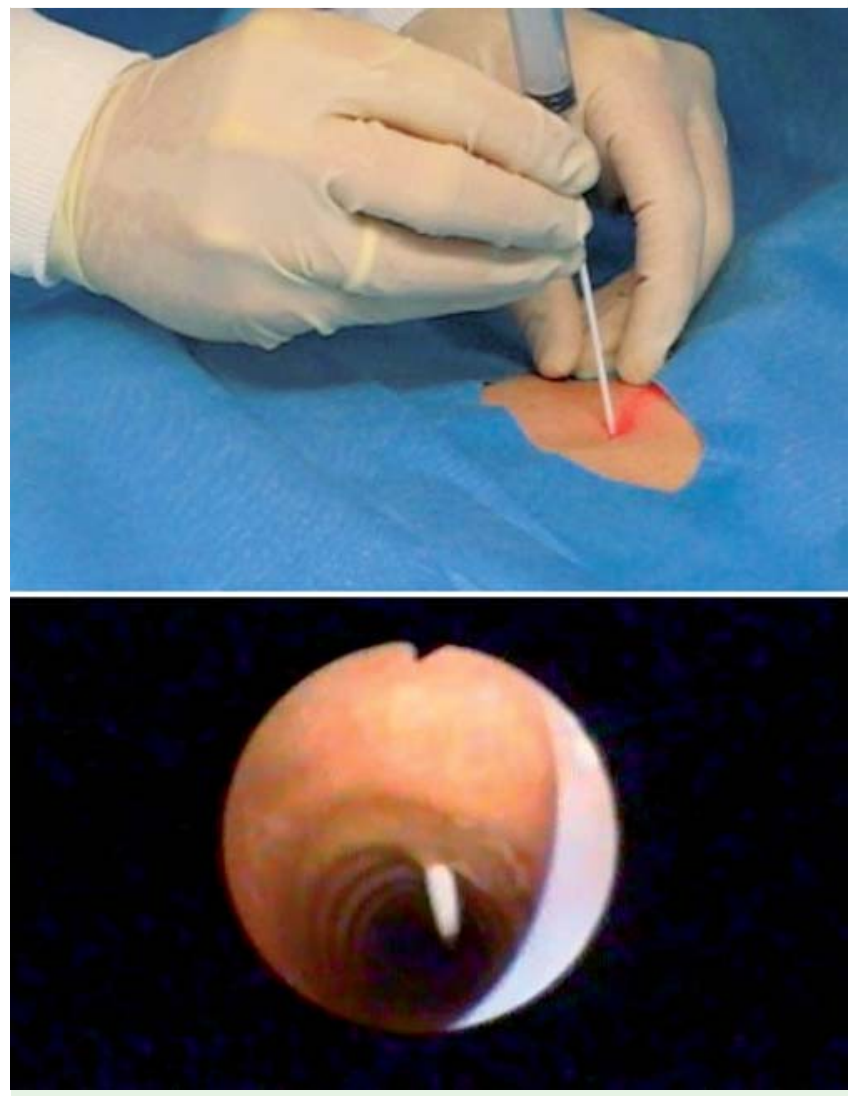

Abb. 2 Perkutane Dilatationstracheotomie. Oben Punktion der Trachea unter Transillumination kaudal der 1. Trachealspange; unten gleichzeitige bronchoskopische Kontrolle des Punktionsvorganges.

\section{Vergleich chirurgische - perkutane Dilatations- tracheotomie}

Komplikationen, und besonders deren relative Häufigkeit, spielen bei der Bewertung der Tracheotomie eine große Rolle. Eine Vielzahl von Studien zum Vergleich der chirurgischen und perkutanen Tracheotomie sowie der unterschiedlichen perkutanen Verfahren untereinander unterstreichen das große Interesse an diesem Thema.

In einer im Jahr 2007 publizierten Metaanalyse [20] wurden 15 randomisierte kontrollierte Studien (insgesamt 973 Patienten) zu diesem Thema verglichen. Es zeigte sich, dass dilatative Tracheotomieverfahren signifikante Vorteile im Hinblick auf die Inzidenz von Stomainfektionen, das kosmetische Ergebnis nach Dekanülierung sowie die Kostenersparnis und die Dauer der Prozedur haben. Die chirurgische Tracheotomie erwies sich als vorteilhaft bezüglich einer geringeren Inzidenz von Kanülendislokationen und -obstruktionen. Bezüglich der Inzidenz von Blutungen, Trachealstenosen und der Gesamtkomplikationsrate inklusive Tod gab es zwischen der chirurgischen und perkutanen Dilatationstracheotomie keine signifikanten Unterschiede.

In Deutschland werden momentan ca. $70 \%$ aller chirurgischen Tracheotomien bei langzeitbeatmeteten Patienten im Operationssaal durchgeführt [19]. Ein Vorteil der dilatativen Technik ist, dass diese Prozedur, unabhängig von OP-Kapazitäten, vom Intensivmediziner selbst bettseitig auf der Intensivstation durchgeführt werden kann. Es entfällt somit der aufwändige und ggf. komplikationsträchtige Transport des Intensivpatienten in den Operationssaal. Zudem ist der zeitliche Aufwand für die perkuta- 
nen Verfahren deutlich geringer als für die offenen. Unter ökonomischen Gesichtspunkten ergibt sich für die dilatative Tracheotomie somit ein Kostenvorteil [21]. Ein weiterer Vorteil der dilatativen Technik ist, dass kein operativer Tracheostomaverschluss erforderlich ist. Sobald die Trachealkanüle nicht mehr benötigt wird, kann diese entfernt und das Stoma abgeklebt werden. Innerhalb von 3-5 Tagen kommt es in der Regel zu einem spontanen Stomaverschluss. Das kosmetische Ergebnis ist bei diesem Verfahren, wie bereits oben erwähnt, deutlich günstiger (- Abb. 3).

Aufgrund der genannten Vorteile wird die perkutane Dilatationstracheotomie heute von den meisten Intensivmedizinern als Verfahren der ersten Wahl angesehen [19].

Im Zusammenhang mit einer evtl. Umstellung der invasiven auf eine nicht-invasive Beatmung ergibt sich ein weiterer Vorteil für die Dilatationstracheotomie. Sofern der Patient nach Entfernung der Trachealkanüle einer nichtinvasiven Beatmung bedarf, kann sich aus einem noch nicht verschlossenen Tracheostoma ein relevantes Problem ergeben. Wird dieses nicht adäquat verschlossen, führt die entstehende Leckage über das Stoma zu einer ineffizienten Beatmung. Pflasterverbände stellen keine Lösung des Leckageproblems dar. Passager kann mit Platzhaltern gearbeitet werden. Problematisch ist die Phase des operativen Stomaverschlusses, da eine Überdruckbeatmung nicht selten zu einer Nahtinsuffizienz und somit zu einem Persistieren der Fistel führt.

\section{Kontraindikationen}

$\nabla$

Generelle Kontraindikationen für chirurgische und dilatative Tracheotomieverfahren sind schwere Weichteilinfektionen im OPGebiet, anatomische Gegebenheiten, die eine Tracheotomie unmöglich machen, und schwerste, nicht korrigierbare Gerinnungsstörungen. Diese Zustände sind selten und gelten sowohl für die chirurgische als auch die perkutane Dilatationstracheotomie. Zusätzlich bestehen aber für die perkutane Dilatationstracheotomie weitere spezielle Kontraindikationen, die im Folgenden erörtert werden sollen.

\section{Absolute Kontraindikationen}

Selbstverständlich stellt das Nichtbeherrschen der notwendigen Technik der perkutanen Dilatationstracheotomie sowie der möglichen Komplikationen eine absolute Kontraindikation dar. Gehört der zu tracheotomierende Patient in ein Risikokollektiv und besteht Unklarheit, ob die Dilatationstracheotomie sicher durchgeführt werden kann, so sollte im Zweifelsfall die chirurgische Tracheotomie vorgezogen werden. Ebenfalls kontraindiziert ist die perkutane Dilatationstracheotomie bei unklaren anatomischen Verhältnissen mit nicht sicher identifizierbarem Trachealverlauf, wie z. B. bei Struma III. Grades oder bei adipösen Patienten mit extrem kurzem Hals.

Weitere absolute Kontraindikationen umfassen erschwerte oder unmögliche Intubationsverhältnisse, eine instabile Halswirbelsäulenfraktur sowie den respiratorischen Notfall. Beim letzteren ist die sofortige endotracheale Intubation, supraglottische Atemwegssicherung oder Koniotomie durchzuführen. Da es bei Kindern aufgrund einer erhöhten Elastizität der Trachea zu Verletzungen der Trachealhinterwand kommen kann, wird in der Regel auch ein jugendliches Alter als Kontraindikation für die Durchführung einer perkutanen Dilatationstracheotomie angesehen.

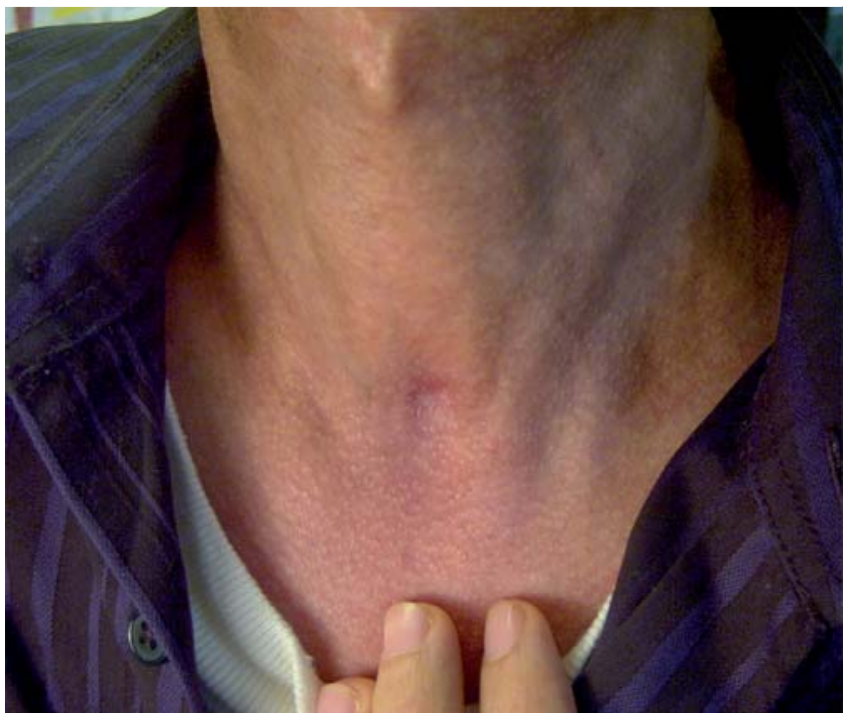

Abb. 3 Kosmetisches Ergebnis nach perkutaner Dilatationstracheotomie bei einem 30-jährigen Patienten (Langzeitbeatmung aufgrund einer Pneumonie nach Stammzelltransplantation).

\section{Relative Kontraindikationen}

Adipositas

Die Lokalisation der anatomischen Orientierungspunkte kann bei ausgeprägter Adipositas erschwert bis unmöglich sein, woraus ein erhöhtes Komplikationsrisiko resultiert [22]. Wenn auch einzelne Autoren über eine im Vergleich zu Normalgewichtigen nicht erhöhte Komplikationsrate berichten [23], sollte unseres Erachtens nach eine Dilatationstracheotomie bei nicht identifizierbarem Trachealverlauf unterbleiben.

\section{Gerinnungsstörungen}

In der Vergangenheit wurden Patienten mit einer Thrombozytopenie oder Gerinnungsstörungen häufig von Studien ausgeschlossen, da diese als Kontraindikationen für das Verfahren gewertet wurden. Neuere Untersuchungen zeigen, dass sowohl

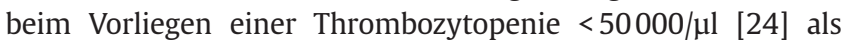
auch bei Koagulopathien auf dem Boden einer Lebererkrankung [25] eine perkutane Dilatationstracheotomie sicher durchgeführt werden kann. Dies ist allerdings nur möglich, wenn neben der vorherigen Substitution von Blut- oder Gerinnungsprodukten, der Eingriff bei diesem Hochrisikokollektiv von einem erfahrenen Team durchgeführt wird. Eine therapeutische Heparinisierung sollte für den Eingriff unterbrochen werden.

\section{Oxygenierungsstörungen}

Eine perkutane Dilatationstracheotomie kann prinzipiell auch bei höhergradig eingeschränktem Gasaustausch und Applikation von hohen PEEP-Werten sicher durchgeführt werden [26]. In der Praxis empfiehlt es sich aber bei schwerem ARDS eine Stabilisierungsphase abzuwarten, da ansonsten nur eine marginale Oxygenierungsreserve bei der Tracheotomie vorhanden ist.

\section{Immunsuppression}

Die Sicherheit der perkutanen Dilatationstracheotomie bei immunsupprimierten Patienten wurde ebenfalls untersucht. In einer eigenen Untersuchung fand sich bei keinem von 51 Patienten, die nach einer Stammzelltransplantation langzeitbeatmet und dilatativ tracheotomiert wurden, eine Stomainfektion [27]. Zwei weitere Publikationen beschreiben die sichere Durchführung von 
perkutanen Dilatationstracheotomien bei fünf [28] und $51 \mathrm{~Pa}-$ tienten [29] nach erfolgter, solider Organtransplantation (Lunge, Herz, Leber, Niere). Stomainfektionen oder andere infektiöse Komplikationen wurden auch bei diesen Patienten nicht beobachtet. Diese Daten zeigen, dass eine perkutane Dilatationstracheotomie auch bei schwerst immunsupprimierten Patienten sicher möglich ist.

\section{Retracheotomie}

Muss ein Patient nach vorangegangener perkutaner Dilatationstracheotomie erneut tracheotomiert werden, so ist bei regelrechten anatomischen Verhältnissen (gut palpabler Trachealverlauf) eine erneute perkutane Dilatationstracheotomie möglich [30].

\section{Außerklinische Beatmung}

Ist die dauerhafte Anlage eines Tracheostomas im Rahmen einer außerklinischen Langzeitbeatmung erforderlich, so muss bei vorhandenem Dilatationsstoma ein sicherer Kanülenwechsel möglich sein. Bei erschwertem Wechsel oder wiederholten Blutungen ist ein Dilatationsstoma in ein chirurgisches Tracheostoma umzuwandeln, um eine sichere Versorgung im außerklinischen Bereich zu ermöglichen [31].

Der Intensivmediziner sollte unbedingt die spezifischen Kontraindikationen der perkutanen Dilatationstracheotomie kennen und beachten. $\mathrm{Zu}$ empfehlen ist in jedem Fall eine Absprache mit der chirurgischen Abteilung, die bei Notfällen nach perkutaner Dilatationstracheotomie zur Verfügung steht. Chirurgische Technik und perkutane Dilatationstracheotomie sind keine konkurrierenden, sondern sich ergänzende Methoden.

\section{Komplikationen}

$\nabla$

Die Inzidenz relevanter Tracheotomie-bedingter Komplikationen ist insgesamt gering. Beschriebene Komplikationen bei der chirurgischen und dilatativen Technik sind u. a. Blutungen, Stomainfektionen, Trachealverletzungen, Pneumothorax, Pneumomediastinum, Hautemphysem, Trachealstenosen, Tracheomalazien und (selten) das Versterben des Patienten. Bei beiden Verfahren besteht zudem, durch den notwendigen Rückzug des Endotrachealtubus, die Gefahr des Atemwegsverlustes.

\section{Blutungen}

Die am häufigsten beobachteten Komplikationen sind Blutungen. Bei der perkutanen Dilatationstracheotomie treten diese im Vergleich zur chirurgischen Tracheotomie etwas seltener auf. Dies ist bedingt durch den kleineren (bis fehlenden) Hautschnitt und eine geringere Gewebetraumatisierung. Geringe Blutungen während der Tracheotomie sistieren meist nach Einsetzen der Trachealkanüle. Hierbei schließt sich das peristomale Gewebe eng um die Trachealkanüle und es kommt zu einer Tamponierung. Selten ist es notwendig kleinere arterielle Blutungen mittels Naht oder Koagulation zu stillen. Sollten während oder nach der Prozedur schwere und/oder anhaltende Blutungen auftreten, die durch obengenannte Maßnahmen nicht beherrscht werden können, so muss eine operative Revision erfolgen.

Eine seltene, aber schwerwiegende postoperative Blutungskomplikation ist die Arrosion des Truncus brachiocephalicus durch die Trachealkanüle oder den Cuff [32]. Hierbei kommt es typischerweise 3-30 Tage nach Tracheotomie zu einer arteriellen Massenblutung ins Bronchialsystem mit konsekutiver Hypoxie. In diesem Fall ist eine notfallmäßige Operation die einzige thera- peutische Option. Als Notfallmaßnahme sollte der Cuff überblockt und die Trachealkanüle auf keinen Fall entfernt oder zurückgezogen werden. Risikofaktoren sind eine zu tiefe (kaudale) Anlage des Stomas, exzessive Halsbewegungen, Zust.n. Radiatio, lange Intubationsdauer und eine Schleimhautschädigung durch Fehllage der Kanülenspitze oder zu hohen Cuffdruck.

\section{Dekanülierung}

Eine problematische Situation stellt die versehentliche Dekanülierung beim frischen dilatativen Stoma dar. Da der Kanal von außen zur Trachea durch das Weichteilgewebe anfangs nur durch die Kanüle selbst offen gehalten wird, kann eine notfallmäßige Rekanülierung schwierig bis unmöglich sein. Wird nun bei geschrumpftem Stoma versucht, die Kanüle mit Gewalt zu reponieren, kann es leicht zu Blutungen, Kanülenfehllagen in die Weichteile und Trachealverletzungen mit Todesfolge kommen. Insbesondere in den ersten 10 Tagen nach perkutaner Dilatationstracheotomie sollte der Patient daher im Notfall primär endotracheal reintubiert und erst in einem zweiten Schritt rekanüliert werden. Dies ist auch einer der Gründe, warum Patienten mit schwierigen Intubationsverhältnissen immer chirurgisch tracheotomiert werden sollten.

Mit zunehmender Zeitdauer nach perkutaner Dilatationstracheotomie formiert sich ein festeres Stoma, ein Trachealkanülenwechsel nach mehr als 10 Tagen stellt im Regelfall für den Geübten kein Problem dar. Der Kanülenwechsel erfolgt am besten in Seldingertechnik über das Bronchoskop oder einen Führungskatheter. Dies sollte gerade in den ersten Tagen möglichst „elektiv“ und am Tage geschehen. Daher sollte jede Pflegekraft und jeder Arzt, der einen tracheotomierten Patienten betreut, die Art des Stomas (dilatativ/chirurgisch) und den Tracheotomiezeitpunkt kennen. In unserer Klinik hat sich das Festnähen der Trachealkanüle nach perkutaner Dilatationstracheotomie bewährt.

Ein Kanülenwechsel nach perkutaner Dilatationstracheotomie sollte in jedem Fall sorgfältig vorbereitet werden und erfordert die Anwesenheit eines erfahrenen Intensivmediziners.

\section{Infektionen}

Stomainfektionen waren in der Vergangenheit ein häufiges Problem und treten bei der Verwendung der perkutanen Dilatationstracheotomie seltener auf. In der 2007 publizierten Metaanalyse wurde diese Komplikation bei 4,8\% der perkutanen Dilatationstracheotomien und bei $12,4 \%$ der konventionellen Tracheotomien beschrieben [20]. Da sich keine erhöhte Rate an prozedurassoziierten Bakteriämien nachweisen ließ, ist eine Antibiotikaprophylaxe nicht erforderlich [33].

\section{Pneumothorax}

Seltene Komplikationen sind die Entwicklung eines Hautemphysems, Pneumothorax oder Pneumomediastinums. Die Inzidenz hierfür liegt bei ca. $1 \%$. Ursächlich können Kanülenfehllagen, Punktionen der Pleura, Verletzungen der Trachealhinterwand oder die Ausbreitung von Luft über den Bougierungskanal in das paratracheale Gewebe sein [34].

Eine Röntgen-Thorax-Kontrolle nach perkutaner Dilatationstracheotomie kann auf komplikationsreiche, technisch schwierige Prozeduren beschränkt werden [35].

\section{Trachealverletzungen/-stenosen}

Verletzungen der Trachealhinterwand sind gefürchtete Komplikationen, die bei der dilatativen Technik häufiger vorkommen, aber auch bei der chirurgischen Tracheotomie beschrieben sind 
[36]. Durch eine sorgfältige bronchoskopische Überwachung während des Dilatationsvorganges kann diese Komplikation weitgehend vermieden bzw. frühzeitig erkannt werden.

In einer der wenigen vergleichenden Studien, in denen auch Langzeitkomplikationen beider Tracheotomieverfahren verglichen wurden, fanden Silvester et al. [37] nach einem Median von 20 Monaten keine erhöhte Inzidenz von Trachealstenosen nach perkutaner Dilatationstracheotomie. Frakturen einzelner Knorpelspangen treten relativ häufig bei der perkutanen Dilatationstracheotomie auf. Denkbar sind dadurch bedingte Reparationsprozesse mit Fibrosierung und Schrumpfung des Gewebes mit nachfolgender Stenosierung der Trachea. Auch bei dieser Patientengruppe fand sich bisher kein Hinweis dafür, dass Trachealspangenfrakturen ein Risikofaktor für die Entwicklung von Trachealstenosen sind [38]. Verletzungen des Ringknorpels, aufgrund einer nicht adäquaten Punktionshöhe, sind im Gegensatz dazu mit einer erhöhten Inzidenz von Trachealstenosen vergesellschaftet [39]. Ein weiteres Argument dafür, dass eine sorgfältige endoskopische Kontrolle als obligat anzusehen ist.

\section{Technische Probleme}

Durch die Punktionsnadel kann es zu Beschädigungen des Tubuscuffs bzw. des Bronchoskops kommen. Ersteres kann bei respiratorisch hochgradig eingeschränkten Patienten einen notfallmäßigen Wechsel des translaryngeal eingebrachten Tubus erforderlich machen, u.U. noch bevor die Tracheotomie abgeschlossen werden kann. Verhindert wird eine Cuffpunktion durch das Zurückziehen des Tubus auf Stimmbandebene. Bevor mit der Nadel punktiert wird, muss das Bronchoskop in den Tubus zurückgezogen werden, um nicht durch die Nadel beschädigt zu werden.

\section{Prophylaxe}

Die Kenntnis der Halsanatomie, die Beachtung der Kontraindikationen, bronchoskopische Kontrolle, gründliche Vorbereitung ( $\mathrm{u}$. a. Material für Notfallintubation am Bett) und ein erfahrenes Team minimieren die Komplikationsrate der Prozedur.

Vor jedem Eingriff muss der vordere Halsbereich sorgfältig inspiziert und palpiert werden. Der Eingriff selbst sollte immer unter bronchoskopischer Kontrolle erfolgen. Sie erlaubt die Bestimmung der Tracheotomiehöhe, idealerweise in der Mittellinie zwischen erster und zweiter bzw. zweiter und dritter Trachealspange [40]. Das endoskopische Bild sollte auch dem Operateur dargestellt werden, da hierdurch die Komplikationsrate reduziert werden kann [41]. Neben der Visualisierung des Punktionsvorgangs können Blutungen in Trachea und Bronchialsystem erkannt und gegebenenfalls Blutkoagel abgesaugt werden. Auf deutschen Intensivstationen wird die Bronchoskopie in 98\% der Fälle routinemäßig eingesetzt [19].

Alternativ zur bronchoskopischen Kontrolle wird in der Literatur eine rein Ultraschall-gestützte Dilatationstechnik beschrieben [42], die unseres Erachtens nicht empfohlen werden kann. Eine sonografische Darstellung der anatomischen Verhältnisse vor der Prozedur kann jedoch im Einzelfall sinnvoll sein, um die Trachea beurteilen und größere bzw. aberrante Gefäße darstellen zu können.

\section{Zeitpunkt der Tracheotomie}

Noch 1989 empfahlen Consensus-Leitlinien eine Tracheotomie, wenn der Patient voraussichtlich $\geq 21$ Tage invasiv beatmet wird [43]. Durch die zunehmende Verbreitung der relativ einfach durchzuführenden, dilatativen Tracheotomieverfahren wird die Indikation zur Tracheotomie heute früher gestellt. 90\% der Patienten in Deutschland werden innerhalb der ersten beiden Wochen nach Beatmungsbeginn tracheotomiert, mit einer Bevorzugung der zweiten Woche [19], dies ist deutlich früher als noch im Jahre 2001 [44].

\section{Tracheotomie versus Weaning mit nichtinvasiver Beatmung (NIV)}

Die Begeisterung über die neuen Tracheotomieverfahren darf den Intensivmediziner nicht dazu verleiten, eine Frühtracheotomie unkritisch bei einem prinzipiell extubierbaren Patienten durchzuführen. Wenn eine Extubation möglich erscheint, so ist dies im Regelfall immer die bessere Alternative. Gerade bei invasiv beatmeten COPD-Patienten ist häufig mit einer schwierigen Entwöhnung zu rechnen. Bei diesem Patientenkollektiv kann durch eine Extubation mit nachfolgender NIV - verglichen mit der invasiv beatmeten Kontrollgruppe - die Erfolgsrate der Respiratorentwöhnung signifikant gesteigert werden. Diesbezüglich liegen mittlerweile die Ergebnisse von drei randomisierten und kontrollierten Studien vor, die eine Senkung von Letalitäts-, Tracheotomie- und Komplikationsrate belegen [45 - 47]. Auch bei Patienten mit ventilatorischer Insuffizienz infolge neuromuskulärer Erkrankungen kann durch die unmittelbar nach der Extubation eingesetzte NIV oftmals die Tracheotomie vermieden werden [48].

Im Gegensatz zur hyperkapnischen Atmungsinsuffizienz bleibt der Stellenwert der NIV allerdings beim schwierigen Weaning infolge hypoxämischer Atmungsinsuffizienz unklar und kann zurzeit nicht empfohlen werden [49].

\section{Studien zur Frühtracheotomie}

Rumbak et al. [11] randomisierten prospektiv 120 internistische Intensivpatienten mit einer zu erwartenden Beatmungsdauer von >14 Tagen zu einer Früh- (Tracheotomie in den ersten 48 Stunden) oder Spättracheotomie (14.-16. Tag). In der Gruppe der frühtracheotomierten Patienten fand sich eine signifikant niedrigere Mortalität ( $32 \%$ vs. $62 \%$ ), eine geringere Rate an Pneumonien ( $5 \%$ vs. $25 \%$ ) und eine kürzere Dauer der Beatmung (7,6 vs. 17,4 Tage)

In der 2008 publizierten französischen Multicenterstudie von Blot et al. [9] wurden 123 überwiegend internistische Intensivpatienten randomisiert zu einer Frühtracheotomie (Tag 1-4) oder zu einer prolongierten Intubation. In den Endpunkten 28-TageMortalität, Inzidenz von nosokomialen Pneumonien, Ventilatorfreie Tage und Intensivverweildauer fand sich kein Vorteil für die Gruppe der Frühtracheotomierten.

In einer aktuell publizierten italienischen Multicenterstudie [50] wurden 419 internistische und chirurgische Patienten zu einer frühen (Tag 6-8) oder späten Tracheotomie (Tag 13-15) randomisiert. Es zeigte sich wiederum keine statistisch signifikante Reduktion von Ventilator-assoziierten Pneumonien in der Frühtracheotomiegruppe. Zudem war die 1-Jahres-Mortalität in beiden Gruppen nicht signifikant different.

Zusammenfassend geben die hier zitierten Studien zur Fragestellung des idealen Tracheotomiezeitpunktes divergente Antworten. $\mathrm{Zu}$ beachten ist die erhebliche Heterogenität der untersuchten Kollektive (z.B. abdominalchirurgisch - neurochirurgisch internistisch) sowie die unterschiedlichen Definitionen des Begriffes „Frühtracheotomie“. So wird eine Frühtracheotomie in einigen Studien als Intervention innerhalb der ersten 2 Tage der invasiven Beatmung definiert, in anderen Studien wird unter 
einer Frühtracheotomie noch eine Tracheotomie bis zum 10. Tag der Beatmung verstanden. Aufgrund der vorliegenden Daten kann die Frühtracheotomie nicht generell für alle Intensivpatienten empfohlen werden. Weitere Untersuchungen, die gezielt die Bedeutung der Frühtracheotomie bei speziellen Kollektiven wie z.B. COPD-Patienten (ClinicalTrials.gov NCT01021202) oder kardiochirurgischen Patienten (ClinicalTrials.gov NCT00347321) evaluieren, werden momentan durchgeführt.

Risiko und Benefit der Prozedur müssen individuell für den einzelnen Patienten abgewogen werden. Bei einer Beatmungsdauer von weniger als 10 Tagen sollte der Patient endotracheal intubiert bleiben. Zur Abschätzung der Beatmungszeit helfen Prädiktoren, wie hohe Krankheitschwere [51], ein niedriger Glasgow Coma Scale Score und das Vorliegen eines ARDS [52]. Kann die Beatmungsdauer nicht adäquat abgeschätzt werden, so sollte die Entscheidung für oder gegen eine Tracheotomie täglich neu bewertet werden.

\section{Fazit}

Die dilatative Tracheotomie ist aufgrund einer Reihe von Vorteilen gegenüber der chirurgischen Tracheotomie zu einem Routineverfahren auf der Intensivstation geworden.

Exakte Kenntnisse der Anatomie, des Operationsverlaufes sowie die Beachtung von Kontraindikationen sind Voraussetzungen für die erfolgreiche Durchführung des Eingriffs.

Zwischen den einzelnen perkutanen Techniken besteht bezüglich der Komplikationsrate kein signifikanter Unterschied. Ob durch eine Frühtracheotomie günstige Effekte erzielt werden können, ist nach der aktuellen Datenlage noch offen.

\section{Interessenkonflikt}

S. Kluge hat eine finanzielle Unterstützung für ein Forschungsvorhaben von der Firma COOK erhalten. H. J. Baumann und C. Kemei geben an, dass kein Interessenkonflikt besteht.

\section{Literatur}

1 Szmuk P, Ezri T, Evron $S$ et al. A brief history of tracheostomy and tracheal intubation, from the Bronze Age to the Space Age. Intensive Care Med 2008; 34: 222-228

2 Walz MK. Die Tracheostomie. Indikationen, Methoden, Risiken. Chirurg 2001; 72: $1101-1110$

3 Cox CE, Carson SS, Holmes GM et al. Increase in tracheostomy for prolonged mechanical ventilation in North Carolina, 1993-2002. Crit Care Med 2004; 32: 2219-2226

4 Griffiths J, Barber VS, Morgan L et al. Systematic review and meta-analysis of studies of the timing of tracheostomy in adult patients undergoing artificial ventilation. BMJ 2005; 330: 1243

5 Ciaglia P, Firsching R, Syniec C. Elective percutaneous dilatational tracheostomy. A new simple bedside procedure; preliminary report. Chest 1985; 87: 715-719

6 Esteller-More E, Ibanez J, Matino E et al. Prognostic factors in laryngotracheal injury following intubation and/or tracheotomy in ICU patients. Eur Arch Otorhinolaryngol 2005; 262: 880-883

7 Diehl JL, El AS, Touchard D et al. Changes in the work of breathing induced by tracheotomy in ventilator-dependent patients. Am J Respir Crit Care Med 1999; 159: 383-388

8 Davis K Jr., Campbell RS, Johannigman JA et al. Changes in respiratory mechanics after tracheostomy. Arch Surg 1999; 134: 59-62

9 Blot F, Similowski T, Trouillet JL et al. Early tracheotomy versus prolonged endotracheal intubation in unselected severely ill ICU patients. Intensive Care Med 2008; 34: 1779- 1787

10 Boles JM, Bion J, Connors A et al. Weaning from mechanical ventilation. Eur Respir J 2007; 29: 1033-1056
11 Rumbak MJ, Newton M, Truncale $T$ et al. A prospective, randomized, study comparing early percutaneous dilational tracheotomy to prolonged translaryngeal intubation (delayed tracheotomy) in critically ill medical patients. Crit Care Med 2004; 32: 1689-1694

12 Sugerman HJ, Wolfe L, Pasquale MD et al. Multicenter, randomized, prospective trial of early tracheostomy. J Trauma 1997; 43: 741 - 747

13 Griggs WM, Worthley LI, Gilligan JE et al. A simple percutaneous tracheostomy technique. Surg Gynecol Obstet 1990; 170: 543 - 545

14 Fantoni A, Ripamonti D. A non-derivative, non-surgical tracheostomy: the translaryngeal method. Intensive Care Med 1997; 23: 386-392

15 Byhahn C, Lischke V, Halbig S et al. Ciaglia Blue Rhino: Ein weiterentwickeltes Verfahren der perkutanen Dilatationstracheotomie. Anaesthesist 2000; 49: 202 - 206

16 Frova G, Quintel M. A new simple method for percutaneous tracheostomy: controlled rotating dilation. A preliminary report. Intensive Care Med 2002; 28: 299-303

17 Zgoda MA, Berger R. Balloon-facilitated percutaneous dilational tracheostomy tube placement: preliminary report of a novel technique. Chest 2005; 128: $3688-3690$

18 Byhahn C, Westphal K, Zwißler B. Die Punktionstracheotomie. Anästh Intensivmed 2005; 46: 125-137

19 Kluge S, Baumann HJ, Maier C et al. Tracheostomy in the intensive care unit: a nationwide survey. Anesth Analg 2008; 107: 1639-1643

20 Higgins KM, Punthakee X. Meta-analysis comparison of open versus percutaneous tracheostomy. Laryngoscope 2007; 117: 447-454

21 Bacchetta MD, Girardi LN, Southard EJ et al. Comparison of open versus bedside percutaneous dilatational tracheostomy in the cardiothoracic surgical patient: outcomes and financial analysis. Ann Thorac Surg 2005; 79: 1879-1885

22 Byhahn C, Lischke V, Meininger D et al. Peri-operative complications during percutaneous tracheostomy in obese patients. Anaesthesia 2005; 60: $12-15$

23 Heyrosa MG, Melniczek DM, Rovito P et al. Percutaneous tracheostomy: a safe procedure in the morbidly obese. J Am Coll Surg 2006; 202 $618-622$

24 Kluge S, Meyer A, Kuhnelt P et al. Percutaneous tracheostomy is safe in patients with severe thrombocytopenia. Chest 2004; 126: 547 - 551

25 Auzinger G, O'Callaghan GP, Bernal W et al. Percutaneous tracheostomy in patients with severe liver disease and a high incidence of refractory coagulopathy: a prospective trial. Crit Care 2007; 11: R110

26 Beiderlinden M, Groeben H, Peters J. Safety of percutaneous dilational tracheostomy in patients ventilated with high positive end-expiratory pressure (PEEP). Intensive Care Med 2003; 29: 944-948

27 Kluge S, Baumann HJ, Nierhaus A et al. Safety of percutaneous dilational tracheostomy in hematopoietic stem cell transplantation recipients requiring long-term mechanical ventilation. J Crit Care 2008; 23: $394-398$

28 Pirat A, Zeyneloglu P, Candan S et al. Percutaneous dilational tracheotomy in solid-organ transplant recipients. Transplant Proc 2004; 36 : $221-223$

29 Waller EA, Aduen JF, Kramer DJ et al. Safety of percutaneous dilatational tracheostomy with direct bronchoscopic guidance for solid organ allograft recipients. Mayo Clin Proc 2007; 82: 1502 - 1508

30 Meyer M, Critchlow J, Mansharamani $N$ et al. Repeat bedside percutaneous dilational tracheostomy is a safe procedure. Crit Care Med 2002; 30: $986-988$

31 Windisch W, Brambring J, Budweiser S et al. Nichtinvasive und invasive Beatmung als Therapie der chronischen respiratorischen Insuffizienz. S2-Leitlinie herausgegeben von der Deutschen Gesellschaft für Pneumologie und Beatmungsmedizin e.V. Pneumologie 2010; 64: 207 240

32 Grant CA, Dempsey G, Harrison J et al. Tracheo-innominate artery fistula after percutaneous tracheostomy: three case reports and a clinical review. Br J Anaesth 2006; 96: 127-131

33 Saayman AG, Findlay GP, Barnes RA et al. Bacteraemia following singlestage percutaneous dilatational tracheostomy. Intensive Care Med 2009; 35: 1970 - 1973

34 Fikkers BG, van Veen JA, Kooloos JG et al. Emphysema and pneumothorax after percutaneous tracheostomy: case reports and an anatomic study. Chest 2004; 125: $1805-1814$

35 Kumar VM, Grant CA, Hughes MW et al. Role of routine chest radiography after percutaneous dilatational tracheostomy. Br J Anaesth 2008; 100: $663-666$

36 Groves DS, Durbin CG Jr. Tracheostomy in the critically ill: indications, timing and techniques. Curr Opin Crit Care 2007; 13: $90-97$ 
37 Silvester W, Goldsmith D, Uchino S et al. Percutaneous versus surgical tracheostomy: A randomized controlled study with long-term followup. Crit Care Med 2006; 34: 2145-2152

38 Higgins D, Bunker N, Kinnear J. Follow-up of patients with tracheal ring fractures secondary to antegrade percutaneous dilational tracheostomy. Eur J Anaesthesiol 2009; 26: 147-149

39 Dollner $R$, Verch $M$, Schweiger $P$ et al. Laryngotracheoscopic findings in long-term follow-up after Griggs tracheostomy. Chest 2002; 122: $206-212$

40 Muhammad JK, Major E, Wood A et al. Percutaneous dilatational tracheostomy: haemorrhagic complications and the vascular anatomy of the anterior neck. A review based on 497 cases. Int J Oral Maxillofac Surg 2000; 29: 217-222

41 Peris A, Linden M, Pellegrini G et al. Percutaneous dilatational tracheostomy: a self-drive control technique with video fiberoptic bronchoscopy reduces perioperative complications. Minerva Anestesiol 2009; 75 : $21-25$

42 Sustic A. Role of ultrasound in the airway management of critically ill patients. Crit Care Med 2007; 35: S173-S177

43 Plummer AL, Gracey DR. Consensus conference on artificial airways in patients receiving mechanical ventilation. Chest 1989; 96: 178-180

44 Westphal K, Byhahn C. Update 2000: Die Tracheotomie in der anästhesiologischen Intensivmedizin. Anaesth Intensivmed 2001; 42: 70-74

45 Girault C, Daudenthun I, Chevron V et al. Noninvasive ventilation as a systematic extubation and weaning technique in acute-on-chronic respiratory failure: a prospective, randomized controlled study. Am J Respir Crit Care Med 1999; 160: 86-92

46 Ferrer M, Esquinas A, Arancibia $F$ et al. Noninvasive ventilation during persistent weaning failure: a randomized controlled trial. Am J Respir Crit Care Med 2003; 168: 70-76

47 Nava S, Ambrosino N, Clini E et al. Noninvasive mechanical ventilation in the weaning of patients with respiratory failure due to chronic obstructive pulmonary disease. A randomized, controlled trial. Ann Intern Med 1998; 128: $721-728$
48 Bach JR, Goncalves MR, Hamdani I et al. Extubation of patients with neuromuscular weakness: a new management paradigm. Chest 2010; 137: $1033-1039$

49 Schönhofer B, Kuhlen R, Neumann P et al. Nichtinvasive Beatmung als Therapie der akuten respiratorischen Insuffizienz. Pneumologie 2008; 62: $449-479$

50 Terragni PP, Antonelli M, Fumagalli R et al. Early vs late tracheotomy for prevention of pneumonia in mechanically ventilated adult ICU patients: a randomized controlled trial. JAMA 2010; 303: 1483-1489

51 Estenssoro E, Gonzalez F, Laffaire E et al. Shock on admission day is the best predictor of prolonged mechanical ventilation in the ICU. Chest 2005; $127: 598-603$

52 King C, Moores LK. Controversies in mechanical ventilation: when should a tracheotomy be placed? Clin Chest Med 2008; 29: 253 - 263,

\section{Bisher erschienene Beiträge dieser Serie}

1 Kelbel C et al. Beatmung und Analgosedierung. Pneumologie 2007; 61: 357-362

2 Kreymann $\mathrm{K}$ et al. Ernährungskonzepte bei Intensivpatienten. Pneumologie 2007; 61: 574-581

3 Hewing $B$ et al. Rationaler Einsatz von Katecholaminen und Inotropika. Pneumologie 2007; 61: 700-709

4 Schild $\mathrm{K}$ et al. Ventilator induzierter Zwerchfellschaden. Pneumologie 2008; 62: 33-39

5 Bein $T$ et al. Extrakorporale Lungenunterstützungsverfahren (ECMO/ iLA). Pneumologie 2008; 62: 137-142

6 Lorenz J et al. Die Therapie der schweren Sepsis. Pneumologie 2009; 63: $197-204$

7 Meyer FJ et al. Herz und Weaning. Pneumologie 2009; 63: 276-281

8 Baumann $\mathrm{HJ}$ et al. Stellenwert der invasiven Diagnostik beim akuten Lungenversagen. Pneumologie 2010; 64: 488-495 